\title{
Aprendizaje-servicio aplicado a los estudios de comunicación
}

\section{José Candón-Mena}

Universidad de Sevilla, España

\section{Resumen}

El proyecto de innovación docente "Red de Educación, Comunicación y Cooperación de la Universidad de Sevilla" (RECCUS) creo una red entre el profesorado de la Universidad de Sevilla y más de una decena de ONG, con fines tanto didácticos como sociales. El proyecto contemplaba diversas acciones basadas en la metodología del aprendizaje-servicio, como la realización de trabajos prácticos mediante los cuales el alumnado elaboró materiales comunicativos para satisfacer necesidades concretas de las entidades participantes. En este texto se detalla la aplicación práctica del proyecto RECCUS siguiendo los elementos clave que deben guiar la implementación del aprendizaje-servicio y se discuten las posibilidades de dicha metodología en el ámbito de la comunicación.

\section{Palabras clave}

Innovación docente, aprendizaje-servicio, comunicación, ONG, tercer sector. 


\title{
Service-learning applied to communication studies
}

\begin{abstract}
The teaching innovation project "Network of Education, Communication and Cooperation of the University of Seville" (RECCUS) created a network between the University of Seville and various NGOs, mainly with didactic and social purposes. The project included several actions such as the preparation of notes and educational videos, visits by communication professionals working for NGOs, the design of a training itinerary and a proposal for a specialized course on communication in the third sector. Its main action, based on the methodology of service-learning, consisted in the realization of practical work through which students developed communication materials to meet the specific needs of the participating entities. This paper details the practical application of the RECCUS project following the key elements that should guide the implementation of the service-learning methodology. The potentialities of this methodology in the field of communication are also discussed.
\end{abstract}

\section{Keywords}

Teaching innovation, service-learning, communication, NGO, third sector. 


\section{Introducción}

La Red de Educación, Comunicación y Cooperación de la Universidad de Sevilla $\left(\right.$ RECCUS $^{1}$ ) es un proyecto de innovación docente basado en el aprendizaje-servicio que involucra al profesorado de las asignaturas de carácter social de la Facultad de Comunicación y a casi una treintena de ONG, con objetivos tanto didácticos como sociales.

El aprendizaje-servicio se define como una "propuesta educativa que combina procesos de aprendizaje y de servicio a la comunidad en un único proyecto bien articulado en el que los participantes aprenden trabajando a partir de necesidades reales del entorno con la finalidad de mejorarlo" (Puig et al., 2007, p.20). Se trata de "una metodología que promueve la adquisición de conocimientos y el servicio a la comunidad, propiciando el desarrollo de competencias sociales y cívicas, y el ejercicio activo de la ciudadanía" (Martínez et al., 2018, p.11).

Bajo las premisas del aprendizajeservicio, los objetivos de RECCUS son mejorar la docencia, la empleabilidad del alumnado, fomentar la concientización sobre temas sociales y el voluntariado en tareas de comunicación en el Tercer Sector.

La Red sirvió para la colaboración docente, compartiendo material o creando otros nuevos (apuntes

\footnotetext{
${ }^{1}$ http://institucional.us.es/reccus
}

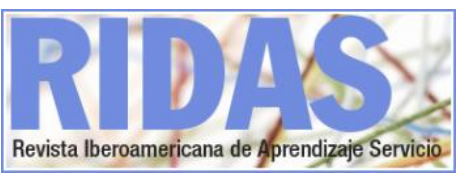

comunes, vídeos didácticos, un itinerario formativo) y creó vínculos con las organizaciones del Tercer Sector, tanto con fines didácticos (prácticas y Trabajos de Fin de Grado (TFG) con casos reales sobre necesidades comunicativas de las entidades, visitas de profesionales de la comunicación en las ONG al aula) y de empleabilidad (prácticas curriculares en ONG), como de Responsabilidad Social Corporativa por parte de la Universidad de Sevilla (captación de voluntariado, difusión de la labor social de la Universidad y de las entidades participantes).

La principal acción, enmarcada en la metodología del aprendizaje-servicio, fueron las "Prácticas prácticas" que consistían en el diseño y planificación de prácticas de clase y TFG sobre casos reales del trabajo comunicativo de las organizaciones del Tercer Sector. Para ello se solicitó a las organizaciones participantes la redacción de una breve propuesta en la que identificaran sus necesidades, que con ayuda del profesorado pudiera convertirse en briefing comunicativo. A partir de dicho briefing, cada uno de los profesores y profesoras diseñó trabajos prácticos adaptados al contenido de sus asignaturas. Para ilustrar esta acción se explicará brevemente el trabajo desarrollado en la asignatura Gabinetes de Comunicación del Grado en Publicidad y RRPP. Tras constituir el aula como una agencia de comunicación de servicios plenos, el alumnado fue dividido en grupos de trabajo para realizar servicios a la ONG local Avanti. Se constituyeron los equipos de: Documentación; Planificación de medios; Estrategia y RR.PP., Creatividad, Redacción, Fotografía, 
Diseño gráfico; e Informática y web. Tras el estudio de las necesidades comunicativas de Avanti y una reunión con el cliente en el aula, se desarrollaron todos los materiales comunicativos de la ONG, entre ellos: Diseño de un nuevo logotipo y un manual de identidad visual corporativa, directorio de medios y mapa de públicos, plan de comunicación y planes específicos para captación de fondos, de socios y de voluntariado, dossier y memoria de actividades de los últimos 5 años y rediseño de su página web. Como resultado los alumnos replicaron la estructura real y tareas de un gabinete de comunicación en el aula en contacto directo con el cliente y la entidad recibió todos los materiales comunicativos necesarios con un acabado profesional y supervisados por el tutor. Por su parte, el alumnado pudo percibir la utilidad real de su trabajo, más allá de la evaluación académica.

\section{Análisis del proyecto RECCUS bajo las premisas del aprendizaje- servicio}

Nos interesa aquí analizar este caso como ejemplo de aprendizaje-servicio para ilustrar el potencial de esta metodología y los pormenores de su aplicación en estudios de comunicación. Para ello tomaremos como referencia los nueve elementos clave que Anna Escofet (2019) señala que debe contener un proyecto de aprendizajeservicio. Nos centraremos fundamentalmente en la acción "Prácticas prácticas" por ser la más específicamente enfocada desde las premisas del aprendizaje-servicio, y en concreto en su implantación en las asignaturas Gabinetes de Comunicación y Publicidad Social del Grado de Publicidad y RRPP.

\subsection{Necesidades sociales}

Según Escofet (2019), éstas deben ser reales, accesibles a las competencias del estudiante y a las posibilidades de hacer alguna aportación, tienen que poder relacionarse con los estudios que cursa el estudiante y su detección debe enmarcarse en un análisis crítico de la realidad y/o de las limitaciones teóricas y prácticas para paliarlas. El

aprendizaje se construye así sobre las necesidades reales del entorno real en el que convive el alumnado.

En el proyecto RECCUS esto se implementa a partir de la definición previa del ámbito de las necesidades a atender, en este caso necesidades estrictamente comunicativas. Dichas necesidades abarcaban no obstante una amplia diversidad, adaptándose a los diferentes grados y asignaturas de la Red, pero siempre dentro del ámbito delimitado de la comunicación. De esta forma se garantizaba la relación del servicio con los estudios del alumnado y sus competencias. Para la detección de estas se pidió a las distintas ONG la elaboración de un documento descriptivo de sus necesidades comunicativas. Este fue posteriormente negociado y definido como un briefing comunicativo con ayuda del profesorado, con el fin de concretar tareas realizables que tuvieran en cuenta las limitaciones temporales y materiales y también respecto a las competencias del alumnado según el grado que estudian, el curso, las asignaturas o las habilidades y 
conocimientos extracurriculares.

\subsection{Partenariado}

En este punto se señala la necesidad de una intensa colaboración entre las entidades y la Universidad para definir conjuntamente los espacios de servicio socialmente útiles, reportar beneficios a las partes y evitar el aprovechamiento inapropiado de ninguna de ellas, situando a las entidades sociales como socios imprescindibles para la realización de la tarea docente (Escofet, 2019).

En particular, consideramos este elemento fundamental para crear una relación recíproca con las entidades en las que exista un equilibrio entre el compromiso exigido con el proyecto y los beneficios recibidos. Para ello el proyecto trató, por una parte, de limitar al mínimo imprescindible las demandas de reuniones, documentación o consultas a las ONG participantes, así como vehicular dichas solicitudes a través del profesorado. En entidades pequeñas y con pocos recursos, que fueron el objetivo prioritario del proyecto, a menudo la buena voluntad de colaboración acaba derivando en una sobrecarga de trabajo para la asociación que resulta contraproducente. Por ejemplo, solicitando documentación, informes, planes de comunicación previos, etc., de los que no disponen al no tener una estructura profesional dedicada a tareas comunicativas, que es de hecho la necesidad que pretende suplirse con el servicio. Desde el proyecto se partió de que incluso la propia identificación de las necesidades, fines u objetivos de la comunicación formaba parte del propio servicio. En general la escasa profesionalización de estas tareas en entidades pequeñas lleva a que haya que partir más bien de intuiciones que requieren del consejo y asesoramiento comunicativo por parte de profesionales, en este caso el profesorado, para dar forma y definir las propias necesidades. Por supuesto ello requiere una negociación conjunta con las entidades para validar las propuestas, pero es fundamental que desde el ámbito profesional de la comunicación se sirva de guía para que la propia entidad tome consciencia del rol que la comunicación puede jugar en el cumplimiento de sus fines sociales, sin crear falsas expectativas.

\subsection{Aprendizaje académico}

Como se ha señalado, el aprendizajeservicio se distingue del voluntariado, las acciones solidarias o el activismo en que debe tener una fuerte base académica, de acuerdo tanto a los contenidos como a las competencias. El servicio debe así ir precedido y seguido por momentos de intensa actividad de aprendizaje (Escofet, 2019). Permite vincular contenidos curriculares y competencias trasversales como el trabajo en equipo, toma de decisiones, liderazgo, entre otras; facilitando la relación entre experiencia y conocimiento. El aprendizaje-servicio ha demostrado ser útil para la adquisición de diversas competencias: personales; interpersonales; para el pensamiento reflexivo, crítico y creativo; para la realización de proyectos; para la ciudadanía y la transformación social; y competencias vocacionales y profesionales (Mayor, 2019; Rubio, 2009). 
El proyecto RECCUS esto se concretó de diversas formas. En primer lugar, los contenidos académicos de la asignatura fueron puestos a disposición del alumnado de forma completa desde el inicio de curso. De esta forma el alumnado disponía de todo el temario y los apuntes de la asignatura, pero en lugar de comenzar con su explicación en el aula por parte del profesorado, se invitaba al alumnado a leer previamente los contenidos. Posteriormente se comenzó a desarrollar el trabajo práctico en clase, intercalando las explicaciones teóricas en los momentos en que éstas podrían ser útiles para el desarrollo del servicio. Se trataba así de hacer patente la intensa relación entre teoría y práctica (Mayor, 2019), y cómo la primera resultaba útil en cada momento concreto para orientar la tarea, estructurarla o ayudar a resolver las dudas planteadas. Por otra parte, el aprendizaje académico se vio reforzado por otras acciones como los apuntes en común o las visitas profesionales y el seminario como actividades académicas complementarias.

\subsection{Enmarcado por la reflexión}

El aprendizaje-servicio, en línea con los modelos de aprendizaje experiencial o de aprendizaje transformativo, otorga gran importancia al proceso de reflexión (Deeley, 2016; Mayor, 2019), programando momentos y ejercicios en los que el estudiante considera su experiencia y toma conciencia personal y crítica del sentido de lo que se ha realizado (Escofet, 2019).

Esta reflexión se materializaba en dos sentidos; sobre las causas sociales y sobre el propio trabajo práctico. La reflexión y toma de conciencia crítica sobre las causas sociales se daba en momentos como la visita de las entidades al aula, en las que antes de describir sus necesidades de servicio se presentaban como organización y exponían las causas sobre las que trabajaban, las visitas profesionales o el seminario, así como en otras actividades complementarias como una visita a la exposición sobre el 'éxodo Rohingya' organizada por Médicos Sin Fronteras. Posteriormente en el transcurso de las clases surgían debates espontáneos sobre temas sociales que eran alentados por el profesorado. Pero también era importante la reflexión crítica sobre el trabajo comunicativo realizado por el alumnado. Por una parte, aunque la exigencia de elaborar materiales con calidad profesional no era requisito imprescindible para aprobar la asignatura, servía para fomentar una autoexigencia por parte del alumnado, que en alguno de los grupos se encontraba en el último curso de la carrera y por tanto a las puertas del mercado laboral, así como para motivarlo y empoderarlo haciéndoles ver cómo su trabajo, aun con ciertos déficits, era muy valorado y resultaba realmente útil para las entidades. El carácter eminentemente práctico de la actividad ayudaba además a que el alumnado percibiera como los conocimientos teóricos adquiridos en sus estudios podían ser aplicados en un caso real y materializarse en un producto final concreto y acabado. Incluso finalizado el curso, muchos alumnos y alumnas se interesaron por la aplicación y el uso efectivo de sus 
trabajos por parte de las ONG y, tras comprobar que efectivamente estaban siendo utilizados, mostraban sentimientos de orgullo y autoestima (Mayor, 2019).

\subsection{Servicio a la comunidad}

La actividad de servicio debe ser socialmente útil, tiene que ser una experiencia rica, no asistencialista y abierta a la reciprocidad. Una actividad no remunerada ni sustitutiva de puestos de trabajo profesionales, tutelada por entidades de marcado e inequívoco carácter social (Escofet, 2019).

Con este punto Escofet advierte, por una parte, de la necesidad de evitar prestar servicios a entidades cuyo carácter social pueda resultar cuestionable, convirtiéndose la actividad en trabajo gratuito en puestos que debieran ser remunerados y organizaciones con fines lucrativos, lo que se evita en el proyecto presentado mediante una criba previa de las organizaciones interesadas en participar para garantizar sus fines netamente sociales. No obstante, esta valoración debe adaptarse a cada caso concreto. Por ejemplo, así ocurrió con la demanda de un servicio para la edición de un vídeo corporativo por parte de la delegación de Bienestar Social del Ayuntamiento de Sevilla, donde la implicación personal de las trabajadoras sociales y de las personas beneficiarias del proyecto, de las que surgía la iniciativa, así como la constatación de la inexistencia de recursos para llevarla a cabo profesionalmente, y por supuesto el carácter no lucrativo de la institución pública, llevó a aceptar la solicitud en este caso concreto. Otro caso similar fue el de un proyecto del Banco del Tiempo gestionado por una asociación vinculada a una empresa lucrativa de animación social por encargo del Ayuntamiento. En este caso también se aceptó la solicitud dado que el servicio se centraba únicamente en el proyecto social, sin vinculación directa con la actividad de la empresa lucrativa que lo gestionaba.

\subsection{Reconocimiento del valor académico y la tarea realizada}

Para Escofet (2019), es imprescindible que la actividad de aprendizaje-servicio sea académicamente exigente y equivalente en esfuerzo y dedicación a cualquier otra actividad docente, conllevando igualmente un reconocimiento para la calificación de la asignatura adecuado al mismo, así como que dicho reconocimiento académico se vincule con el aprendizaje y no como recompensa por el servicio prestado.

Aunque la calificación de la actividad fue variable en cada asignatura del proyecto RECCUS, todo el profesorado implicado se comprometió a incluir la actividad en los criterios de calificación, ya fuera como porcentaje de la nota final o como prácticas voluntarias para obtener puntos extra. En el caso de las asignaturas más vinculadas con la comunicación social, la actividad llegaba a suponer más del $80 \%$ de la nota final, valorándose además la asistencia a actividades como las visitas profesionales o el seminario con hasta dos puntos extra, de forma que en conjunto podían sustituir al examen, dado que los contenidos teóricos se 
asimilaban de forma paralela al diseño y ejecución del servicio. Sin embargo, este método de calificación no supuso una merma de la exigencia, dándose el caso de grupos de trabajo que de hecho suspendieron la actividad.

\subsection{Participación y trabajo en grupo}

En primer lugar, el trabajo en grupo fue un requisito fundamental para la realización de la actividad, tanto por exigencias organizativas como por el propio valor de trabajar en colectividad, no solo por los valores sociales como el desarrollo de habilidades cooperativas como el diálogo, la empatía, la asertividad o la negociación en busca del consenso (Picazo et al., 2009), sino también por su utilidad didáctica para acercar al alumnado a la realidad profesional.

El proyecto se orientó desde el inicio para promover la participación activa y la involucración en la toma de decisiones del alumnado, fomentando el trabajo autónomo y apoyado en las tutorías grupales. En primer lugar, el alumnado fue el encargado de decidir cuáles serían los proyectos seleccionados entre aquellos presentados por las ONG, de forma que ellos mismos eligieron por consenso los temas sobre los que trabajarían en clase y las entidades con las que colaborarían, lo que lógicamente influiría en una mayor motivación con el servicio. Pero, además, el alumnado eligió también la cantidad y funciones de los grupos de trabajo y en cuáles participaría cada uno, con el único criterio de un máximo de miembros y la orientación sobre las tareas esenciales por parte del profesor. De esta forma la clase se constituyó como una agencia de comunicación con distintos departamentos decidiendo cada alumno tanto la división general en grupos de trabajo como los grupos en los que involucrarse, lo que permitía que se unieran según intereses y competencias afines.

En definitiva, el alumnado fue protagonista de la toma de decisiones desde la fase de organización y diseño de la actividad hasta el momento del desarrollo. Finalmente, la necesaria coordinación entre grupos e incluso la dependencia funcional de tareas que requerían partir del trabajo previo de otros grupos fomentaba la colaboración general al nivel de toda la clase.

\subsection{Evaluación}

En cuanto a la evaluación del proyecto, se realizó una encuesta propia que fue complementada con la encuesta realizada por la Oficina de Cooperación al Desarrollo con motivo del seminario organizado conjuntamente. También se evaluó la asistencia a distintas actividades. La captación de voluntariado entre el alumnado por parte de las ONG participantes supone también un aspecto importante de la evaluación y uno de los resultados más destacables en el plano social. Los resultados de dichas evaluaciones se detallarán en el siguiente apartado.

\subsection{Consolidación}

Surgida por la convocatoria de un proyecto de innovación docente, la constitución como red de RECCUS mostraba el ánimo de consolidación de un proyecto estable en el tiempo. Cabe 
destacar que el proyecto tiene como antecedente acciones de aprendizajeservicio puntuales que ya venían realizando algunos profesores de la Facultad y que se formalizan con la creación de RECCUS en el curso 20172018. Sin embargo, acabada la convocatoria formal, la red continúa activa realizando actividades de aprendizaje-servicio e incorporando a nuevas ONG. Fruto de los contactos mantenidos, el proyecto será además incorporado a un nuevo proyecto de innovación docente y otro de cooperación al desarrollo sobre aprendizaje-servicio presentado por profesoras de la Facultad de Educación, con las que a raíz del proyecto inicial y jornadas sobre aprendizaje-servicio se ha venido colaborando.

\section{Evaluación y resultados}

La evaluación del proyecto por parte del alumnado se realizó por medio de encuestas, así como de la asistencia y participación en las actividades complementarias.

Por una parte, se realizaron encuestas propias en dos grupos de clase (109 en total) usando un cuestionario tipo test con escala de Likert para valorar el cumplimiento tanto de los objetivos didácticos como de los sociales.

Respecto a los primeros, el $76,15 \%$ del alumnado se mostró de acuerdo/totalmente de acuerdo con la afirmación de que había mejorado su conocimiento sobre la práctica real de la comunicación en el mundo profesional $(2,75 \%$ en desacuerdo/muy en desacuerdo, el resto ni de acuerdo ni en desacuerdo), el 73,15\% consideró que había aprendido más gracias al proyecto $(2,75 \%$ en desacuerdo), un $60,48 \%$ que el mismo le había facilitado una formación especializada en comunicación social $(4,59 \%$ en desacuerdo), el 59,63\% consideraba que el proyecto podría ayudarle a encontrar empleo en el futuro $(14,68 \%$ en desacuerdo) y para un $50,74 \%$ las clases le habían resultado más interesantes $(18,69 \%$ en desacuerdo).

En cuanto a los objetivos sociales, un $62,28 \%$ del alumnado afirmó que se planteaba colaborar como voluntario en alguna organización social, un 49,09\% realizar una donación, un $48,15 \%$ realizar prácticas curriculares en entidades sociales y un $45,45 \%$ hacerse socio de alguna ONG. Además, para el $74,04 \%$ había mejorado el conocimiento de las organizaciones y causas sociales del Tercer Sector.

Se evaluó también la acogida de distintas actividades, resultando que un $82,57 \%$ consideró interesantes/muy interesantes el conjunto de las visitas de profesionales a clase, un $80,77 \%$ valoró positivamente el Seminario y un $75 \%$ consideró de interés o mucho interés la grabación de programas en Radiopolis. Pero la actividad mejor valorada fue con diferencia la de las "Prácticas prácticas", que implicaba directamente el aprendizaje-servicio, considerada como interesante o muy interesante por el 96,37\% del alumnado participante, de los que el $40 \%$ las consideraban muy interesantes, quedando solo un 3,64\% de indiferentes y sin respuestas negativas. 


\section{Conclusiones}

La experiencia de la Red ha servido para fortalecer lazos entre el profesorado y las ONG, llegándose incluso a recibir múltiples peticiones de otras entidades interesadas en sumarse al proyecto. Los objetivos didácticos se han cumplido teniendo una gran aceptación por parte del alumnado. La escasez de prácticas y la excesiva carga teórica es una de las quejas más habituales entre el alumnado de la Facultad por lo que esta experiencia ha sido muy bien valorada. En cuanto a los objetivos sociales, muchas entidades han recibido materiales comunicativos para satisfacer las necesidades que plantearon. Además, han sido varios los alumnos que han comenzado a colaborar como voluntarios en las entidades participantes o que, tras la experiencia, han decidido realizar sus TFG en colaboración con las mismas.

Consideramos que el aprendizajeservicio resulta especialmente adecuado para las enseñanzas en comunicación ya que se trata de un servicio útil para todo tipo de entidades. Por una parte, en entidades pequeñas que rara vez disponen de un departamento o profesionales dedicados a estas tareas, las carencias comunicativas abren un amplio abanico de servicios que pueden realizarse de forma satisfactoria por parte del alumnado en formación, suponiendo una mejora cualitativa para la entidad. Se trata además de un servicio muy adaptable a las distintas áreas de estudio, que puede abarcar tareas propias del periodismo, la publicidad o la comunicación audiovisual, y también escalable, permitiendo trabajar sobre proyectos pequeños como un cartel, un folleto o un breve dossier o tareas más complejas como un plan de comunicación, una memoria o producciones audiovisuales.

\section{Referencias bibliográficas}

Deeley, S. (2016). Aprendizaje-servicio en educación superior. Teoría, práctica y perspectiva crítica. Madrid, España: Narcea.

Escofet, A. (Abril de 2019). La metodología del aprendizaje-servicio como respuesta a los retos de la sociedad desde las aulas universitarias [Actividad de formación]. Jornada de Formación Aprendizaje-Servicio en el ámbito universitario. Facultad de Ciencias de la Educación, Universidad de Sevilla, España.

Martínez, V., Melero, N., Ibáñez, E. y Sánchez, M. (Eds.) (2018). El Aprendizaje-Servicio en la Universidad. Una metodología docente y de investigación al servicio de la justicia social y el desarrollo sostenible. Salamanca, España: Comunicación Social Ediciones y Publicaciones.

Mayor, D. (2019). El aprendizajeservicio como eje articulador de procesos de desarrollo personalestudiantil y social en el estudiantado universitario. Perfiles Educativos, 41(166), 124-140. Recuperado de http://perfileseducativos.unam.mx/iisu e pe/index.php/perfiles/article/view/58 $\underline{835 / 52333}$

Picazo, C., Zornoza, A. y Peiró, J. M. (2009). Los procesos de participación social y participación orientada a la 
tarea y el aprendizaje como

antecedentes de la cohesión grupal.

Una perspectiva longitudinal.

Psicothema, 21(2), 274-279.

Recuperado de

http://www.psicothema.com/pdf/3626.

pdf

Puig, J. M., Batlle, R., Bosch, C. y Palos,

J. (2007). Aprendizaje servicio. Educar

para la ciudadanía. Barcelona, España:

Octaedro.

Rubio, L. (2009). El aprendizaje en el aprendizaje servicio. En J. M. Puig

(Coord.). Aprendizaje servicio.

Educación y compromiso cívico (pp. 91106). Barcelona, España: Graó. 